Article

\title{
Investigation on the Rapid Annealing of Ti-Au Composite Electrode on n-Type (111) CdZnTe Crystals
}

\author{
Haozhi Shi, Shulei Wang, Jijun Zhang *, Zhubin Shi, Jiahua Min, Jian Huang and Linjun Wang \\ Department of Electronic Information Materials, School of Materials Science and Engineering, Shanghai \\ University, Shanghai 200444, China; shihaozhi@shu.edu.cn (H.S.); CYTTT@shu.edu.cn (S.W.); \\ shizhubin@shu.edu.cn (Z.S.); minjh@mail.shu.edu.cn (J.M.); jianhuang@shu.edu.cn (J.H.); \\ ljwang@shu.edu.cn (L.W.) \\ * Correspondence: zhangjijun81@shu.edu.cn; Tel.: +86-029-6613-6126
}

Received: 26 December 2019; Accepted: 26 February 2020; Published: 29 February 2020

\begin{abstract}
In this paper, the ohmic properties of $\mathrm{Ti}, \mathrm{Al}$, and Ti-Au composite electrodes on n-type (111) CdZnTe crystal deposited by vacuum evaporation method were first analyzed, and then the rapid annealing of Ti-Au electrode under Ar atmosphere with different temperature and time was explored. The ohmic property and barrier height were evaluated by current-voltage (I-V) and capacitance-voltage $(\mathrm{C}-\mathrm{V})$ measurements, and the adhesion strength of various electrodess to CdZnTe was compared. The Ti-Au electrode on CdZnTe showed the lowest leakage current and barrier height, and the highest adhesion strength among the three kinds of electrodes on (111) CdZnTe crystals. The rapid annealing of Ti-Au electrode under Ar atmosphere was proved to improve its ohmic property and adhesion strength, and the optimal annealing temperature and time were found to be $423 \mathrm{~K}$ and $6 \mathrm{~min}$, respectively. The barrier height of the Ti-Au/CdZnTe electrode is $0.801 \mathrm{eV}$ through rapid annealing for $6 \mathrm{~min}$ at $423 \mathrm{~K}$ annealing temperature, and the adhesion is $1225 \mathrm{MPa}$, which increases by $50 \%$ compared with that without rapid annealing.
\end{abstract}

Keywords: CdZnTe; Ti-Au composite electrode; ohmic property; adhesion strength; rapid annealing

\section{Introduction}

CdZnTe nuclear radiation detector can work at room temperature, with high detection efficiency and good energy resolution for X-ray and gamma rays [1-8]. The preparation of CdZnTe nuclear radiation detector with good performance is not only affected by the crystal quality of CdZnTe crystal, but also associated with the contact of electrode. The electrode can directly affect the electrical performance of the device, and the leakage current, electric field distribution, and carrier transport of the semiconductor device are closely related to the electrode $[9,10]$.

At present, the contact performance between CdZnTe crystal and the electrode is mainly improved by the selection of electrode materials, surface treatment, and annealing process after the preparation of the electrode. Sun et al. [11] observed the crystal surface of CdZnTe by scanning electron microscopy and transmission electron microscopy. The results showed that there was a $9.2 \%$ lattice mismatch between $\mathrm{Au}$ atom and Te after chemical polishing. Tari et al. [12] studied the element composition of the interface between CdZnTe and Au electrodes by X-ray Photoelectron Spectroscopy (XPS). It was found that, when CdZnTe crystals were exposed to atmospheric environment for few hours, a native oxide layer formed on the surface of CdZnTe, which was harmful to the electrical properties of CdZnTe crystals. Yang et al. [13] found that evaporating Au-Zn composite electrodes on the (111) surface of CdZnTe crystal can effectively avoid the formation of an oxide layer. Ling et al. [14] indicated that the Au-Cd composite electrode evaporated on p-CdZnTe (111) plane can obtain better ohmic contact performance with a lower barrier height than the $\mathrm{Au}-\mathrm{Zn}$ electrode. The adhesion between metal 
electrode and CdZnTe crystal is important for the detector fabrication process. Bell et al. [15] found that the electrode may adhere to the photoresist during the fabrication of pixel CdZnTe detectors, which causes the electrode to be lost before lithography.

In this paper, $\mathrm{Ti}, \mathrm{Al}$, and $\mathrm{Ti}-\mathrm{Au}$ electrodes were selected to deposit on the surface of n-type (111) CdZnTe crystals by the vacuum evaporation method. The electric properties of the three kinds of electrodes are evaluated by current-voltage (I-V) measurement, and the potential barrier height between the three electrodes and the crystal interface is calculated by capacitance-voltage $(\mathrm{C}-\mathrm{V})$ measurement. The characteristics of the Ti-Au composite electrode through different annealing processes are analyzed. The results show that the best ohmic characteristics and adhesion are obtained after $6 \mathrm{~min}$ rapid annealing at $423 \mathrm{~K}$ annealing temperature.

\section{Experimental}

\subsection{Electrodes Depositions}

The In-doped CdZnTe crystals were grown by the vertical Bridgman method with n-type conductivity. For the ohmic contact of n-type CdZnTe crystal, according to the metal-semiconductor contact principle, metal with a small work function was selected to form the n-type anti-barrier layer between the metal and semiconductor. The work functions of metal Al and Ti are smaller than n-type CdZnTe [16]. Therefore, the $\mathrm{Al}$ and Ti electrodes were chosen to study the ohmic property with n-type CdZnTe crystals. In order to decrease the low thermal stability and oxidation effect of Ti electrodes, the Au film was deposited on the Ti electrode to form the Ti-Au composite electrode on CdZnTe crystal.

Three adjacent n-type (111) CdZnTe crystals with dimensions of $10 \times 10 \times 2 \mathrm{~mm}^{3}$ were used to prepare $\mathrm{Ti}, \mathrm{Al}$, and Ti-Au metal electrodes. The crystals were chemically and mechanically polished by a Logitech PM6 polisher. Then, the metal electrodes were deposited on CdZnTe crystals by the vacuum evaporation method with MBE-600. During the evaporation process, the vacuum degree was higher than $2 \times 10^{-3} \mathrm{~Pa}$, the current was lower than $130 \mathrm{~A}$, and the evaporation time was controlled at about $10 \mathrm{~min}$.

The Ti-Au composite electrode was prepared by depositing Ti layer on the chemical mechanical polished CdZnTe crystal, and then depositing Au on the Ti layer. The Ti-Au composite electrode was then held for 30 minutes to promote the alloying process. The I-V and C-V electrical performance test instruments used were as follows: Keithley 4200SCS (Shanghai, China) semiconductor property analysis system, with a bias range of $-20 \sim 20 \mathrm{~V}$ and a minimum step size of $0.1 \mathrm{~V}$; HP4140B micro-current tester (Agilent Tech, Shanghai, China), with a measuring range of $10^{-5}-10^{-2} \mathrm{~A}$ and a maximum resolution of $10^{-15} \mathrm{~A}$.

\subsection{Annealing of Ti-Au/CdZnTe Electrode}

The Ti-Au/CdZnTe composite electrode was deposited on the surface of n-CdZnTe crystal by the vacuum evaporation method. Then, the crystals were placed in AG610, which is an atmosphere annealing furnace. The effects of annealing temperature and annealing time on the contact properties of Ti-Au/CdZnTe were studied under an argon (Ar) atmosphere.

Firstly, the annealing temperature was selected to be $373 \mathrm{~K}, 423 \mathrm{~K}, 473 \mathrm{~K}, 523 \mathrm{~K}$, and $573 \mathrm{~K}$, respectively, and the Ti-Au/CdZnTe electrode was annealed for 4 min to determine the optimal annealing temperature parameters. Secondly, the Ti-Au/CdZnTe electrode was rapidly annealed for $2 \mathrm{~min}, 4 \mathrm{~min}, 6 \mathrm{~min}, 8 \mathrm{~min}$, and $10 \mathrm{~min}$ at the optimal temperature, respectively. In the process of rapid annealing, the temperature was rapidly raised to the target annealing temperature in 30 seconds, and then the annealing was conducted according to the set annealing time. After the annealing, the temperature was rapidly cooled to room temperature. 


\section{Results and Discussions}

\subsection{Comparison of Ti, Al, and Ti-Au Electrodes to CdZnTe Crystals}

Figure 1 lists the $\mathrm{I}-\mathrm{V}$ characteristic curves of $\mathrm{Ti}, \mathrm{Al}$, and $\mathrm{Ti}-\mathrm{Au}$ composite electrodes, which are fitted according to the ohmic characteristic formula $I(V)=a V^{b}$, where $a$ is a constant, $b$ is the ohmic coefficient, and $b=1$ is the ideal ohmic contact. The fitting results are shown in Table 1 . It can be seen that the ohmic characteristics of $\mathrm{Al}$ electrode are poor, and the leakage current at $20 \mathrm{~V}$ is the highest. The ohmic coefficient of Ti-Au composite electrode is the closest to 1, indicating that the ohmic characteristic is good and the leakage current is the lowest.

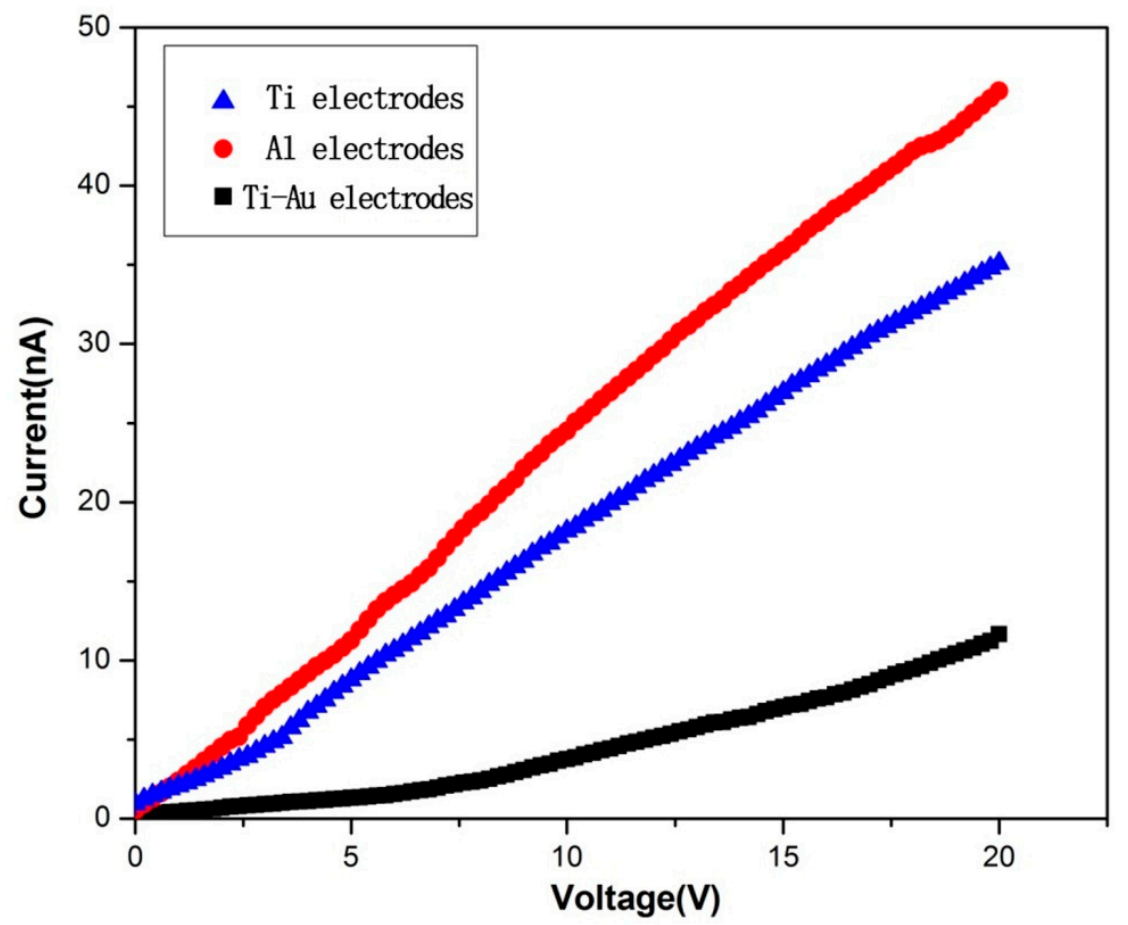

Figure 1. The current-voltage (I-V) characteristic curves of $\mathrm{Ti}, \mathrm{Al}$, and $\mathrm{Ti}-\mathrm{Au}$ electrodes to (111) CdZnTe crystals.

Table 1. The current-voltage (I-V) curves fitting results of Ti, Al, and Ti-Au electrodes to n-type (111) CdZnTe crystals.

\begin{tabular}{cccc}
\hline Electrode Type & Ti & Al & Ti/Au \\
\hline Ohmic coefficient b & 0.7511 & 0.6763 & 0.8543 \\
leakage current nA@20 V & 35.12 & 45.93 & 11.67 \\
\hline
\end{tabular}

The $\mathrm{C}-\mathrm{V}$ measurement was carried out on $\mathrm{Ti}, \mathrm{Al}$, and $\mathrm{Ti}-\mathrm{Au}$ electrodes with the test frequency of $1 \mathrm{MHz}$, and the results are shown in Figure 2. It can be seen that the change trend of capacitance $\mathrm{C}$ of Ti-Au/CdZnTe with applied voltage is the most obvious, and its carrier concentration is the highest. The CdZnTe detector acts as a parallel plate capacitor. When the detector is applied with a positive voltage, electrons will enter the metal from the semiconductor, and the reverse current will increase. The hole concentration in the space charge region will decrease compared with the equilibrium. To achieve equilibrium, the width of the space charge region will increase continuously, and the capacitance $C$ will decrease until the device is completely exhausted. Therefore, the carrier concentration can be determined by comparing the curve of capacitance $C$ with applied voltage V [17]. 


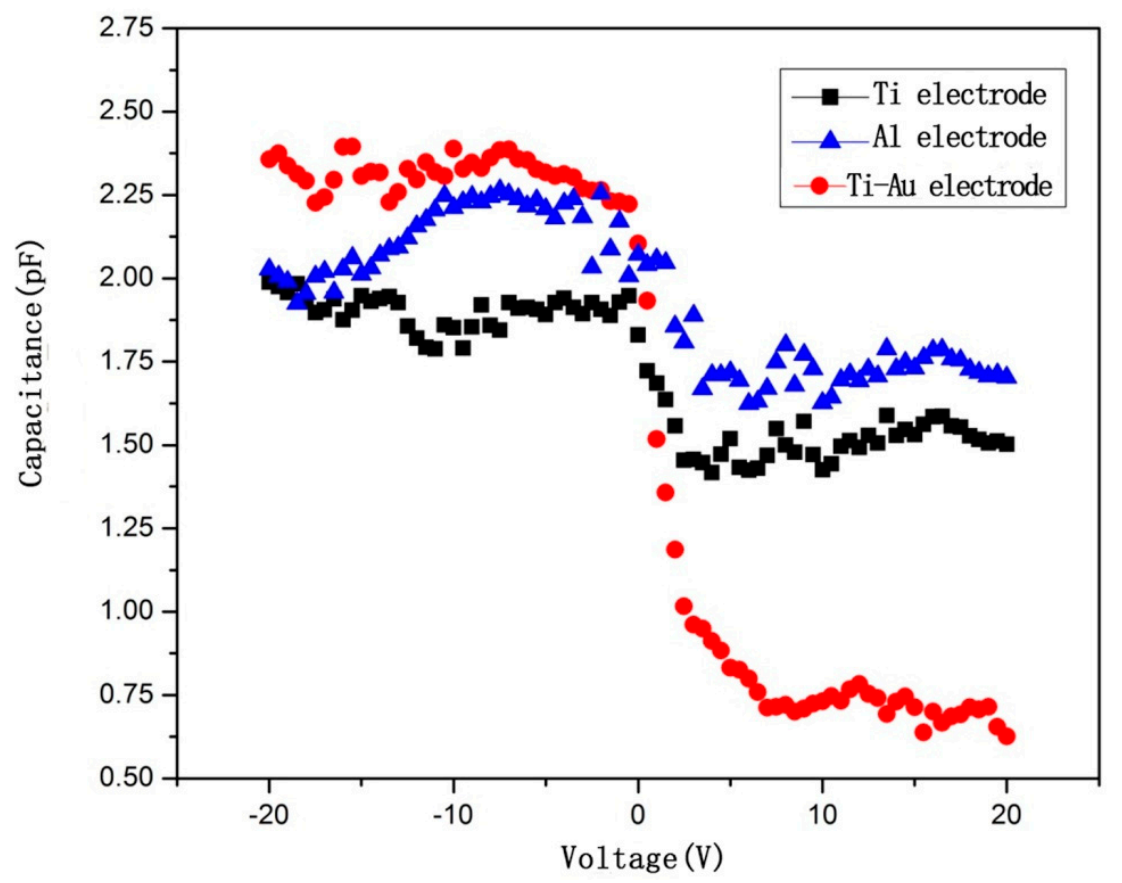

Figure 2. The capacitance-voltage (C-V) characteristic curves of $\mathrm{Ti}, \mathrm{Al}$, and Ti-Au electrodes to (111) CdZnTe crystals.

According to the $\mathrm{C}-\mathrm{V}$ test, the barrier height $\Phi_{B}$ can be calculated as follows:

$$
\Phi_{B}=V_{\mathrm{int}}+k T / q k T \ln \left(N_{V} / N_{A}\right) / q
$$

where $V_{\text {int }}$ is the intercept of the characteristic on axis $\mathrm{V} ; q$ is the electron charge; and $N_{A}$ and $N_{V}$ are the acceptor impurity concentration and valence band effective state density, respectively, which are calculated by the following equations:

$$
\begin{gathered}
N_{A}=\frac{2}{q \varepsilon_{0} \varepsilon_{s}}\left\{\frac{-d V}{d C^{-2}}\right\} \\
N_{V}=2 \times 10^{-6} \cdot\left(2 \pi m_{p} k T / h^{2}\right)^{3 / 2}
\end{gathered}
$$

where $\varepsilon_{s}$ is the relative dielectric constant of crystal, $\varepsilon_{0}$ is the vacuum dielectric constant, $V$ is the external voltage, and $C$ is the junction capacitance. The calculation results are shown in Table 2. The barrier height of $\mathrm{Al} / \mathrm{CdZnTe}$ is lower than that of Ti/CdZnTe. This may because of the higher diffusion of $\mathrm{Al}$ through the interface compared with Ti in CdZnTe, which lowers the barrier height of $\mathrm{Al} / \mathrm{CdZnTe}$, but also has the effect of increasing the leakage current, as shown in Figure 1. The Ti-Au/CdZnTe sample has the smallest barrier height, which indicates that the Ti-Au electrode can form better ohmic contact with n-type (111) CdZnTe crystalthan the other two electrodes. The barrier height is determined by the work functions of the metal and semiconductor and the presence of surface states. Owing to the low thermal stability of $\mathrm{Al}$ and Ti metal electrodes, oxidation will occur during the evaporation process, resulting in a rough electrode surface and formation of oxides, leading to degradation of ohmic contact properties. As the Au electrode is not easily oxidized, it is assumed that the Ti-Au composite electrode formed by Au coverage on Ti has the effect of decreasing the surface state, which favours the ohmic property. 
Table 2. The barrier heights of Ti, Al, and Ti-Au electrodes to n-type (111) CdZnTe crystals.

\begin{tabular}{cc}
\hline Electrodes & $\boldsymbol{\Phi}_{\mathbf{B}}(\mathbf{e V})$ \\
\hline $\mathrm{Ti}$ & 0.975 \\
$\mathrm{Al}$ & 0.962 \\
$\mathrm{Ti}-\mathrm{Au}$ & 0.928 \\
\hline
\end{tabular}

The adhesion strength between the $\mathrm{Ti}, \mathrm{Al}$, and Ti-Au electrodes and $\mathrm{CdZnTe}$ crystals was measured by a Dage PC 2400 microanalyzer. This experiment is destructive on contact electrodes by the shear-off method, which is generally used to measure the adhesion of two contact objects [18]. The test principle is shown in Figure 3.

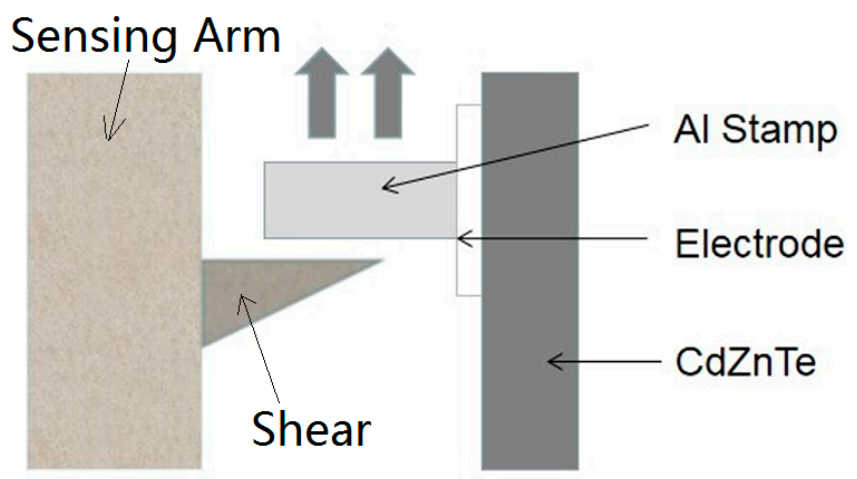

Figure 3. The schematic diagram of the film adhesion test.

The adhesion strength between the electrodes and CdZnTe crystals was calculated by the thrust (F) and the adhesion area (A) of the Al block. The formula is as follows:

$$
\sigma_{\text {shear }}=F / A
$$

The adhesion strength between the $\mathrm{Ti}, \mathrm{Al}$, and $\mathrm{Ti}-\mathrm{Au}$ electrodes and $\mathrm{CdZnTe}$ crystals was measured three times, and the average values were taken, which are shown in Figure 4 . It can be seen that the adhesion between the Ti-Au electrode and CdZnTe crystal is significantly higher than that of the Ti and Al electrode, with the average adhesion strength of 835.3 Mpa.

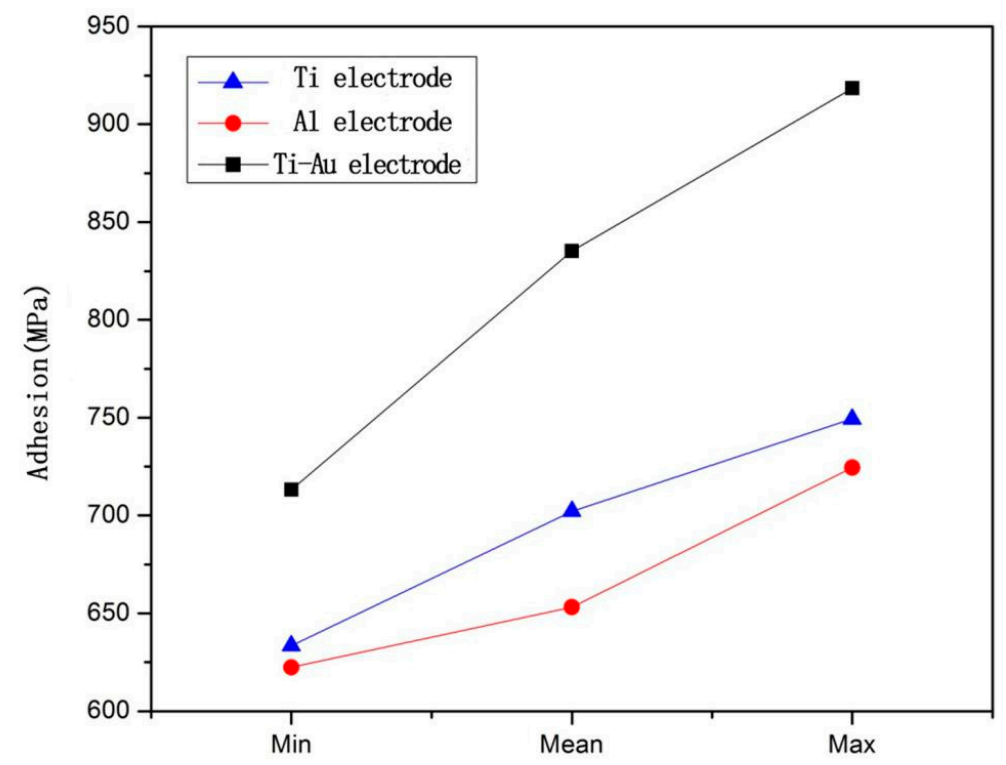

Figure 4. The adhesion between the $\mathrm{Ti}, \mathrm{Al}$, and Ti-Au electrodes and $\mathrm{CdZnTe}$ crystals. 


\subsection{Rapid Annealing of Ti-Au/CdZnTe}

\subsubsection{Effect of Annealing Temperature on Ti-Au/CdZnTe}

Five n-type CdZnTe crystals deposited with Ti-Au composite electrode are used for rapid annealing experiments at different annealing temperatures $(373 \mathrm{~K}, 423 \mathrm{~K}, 473 \mathrm{~K}, 523 \mathrm{~K}$, and $573 \mathrm{~K})$ for 4 min. The effect of annealing temperature on the Ti-Au/CdZnTe electrode is first studied by I-V measurements, as shown in Figure 5. The fitting results of I-V curves are shown in Table 3. The results indicate that, with the increase of annealing temperature from no annealing to $423 \mathrm{~K}$, the leakage current of the $\mathrm{Ti}-\mathrm{Au} / \mathrm{CdZnTe}$ sample decreases. When the annealing temperature is $423 \mathrm{~K}$, the leakage current is the lowest. When the temperature continues to increase, however, the leakage current rises sharply. The results of the ohmic characteristic with different annealing temperature revealed the same trend, as the annealing temperature is $423 \mathrm{~K}$, the ohmic coefficient $\mathrm{b}$ is closer to 1 . When the annealing temperature is higher than $573 \mathrm{~K}$, the ohmic contact property of the electrode decreases, and the leakage current is the highest. In the initial process of rapid annealing, the leakage current decreases and the ohmic contact performance improves, mainly because the annealing treatment reduces defects in the crystal, increases alloying of Ti-Au composite electrode, and finally forms a diffusion layer in the interface between the electrode and CdZnTe crystal [19]. However, when the temperature is further increased, the diffusion layer penetrates to the depth of the crystal, which leads to the increase of leakage current. Meanwhile, the high annealing temperature also reduces the uniformity of electrode surface and interface. Therefore, a suitable rapid annealing temperature with $423 \mathrm{~K}$ can effectively improve the ohmic property of the Ti-Au/CdZnTe sample.

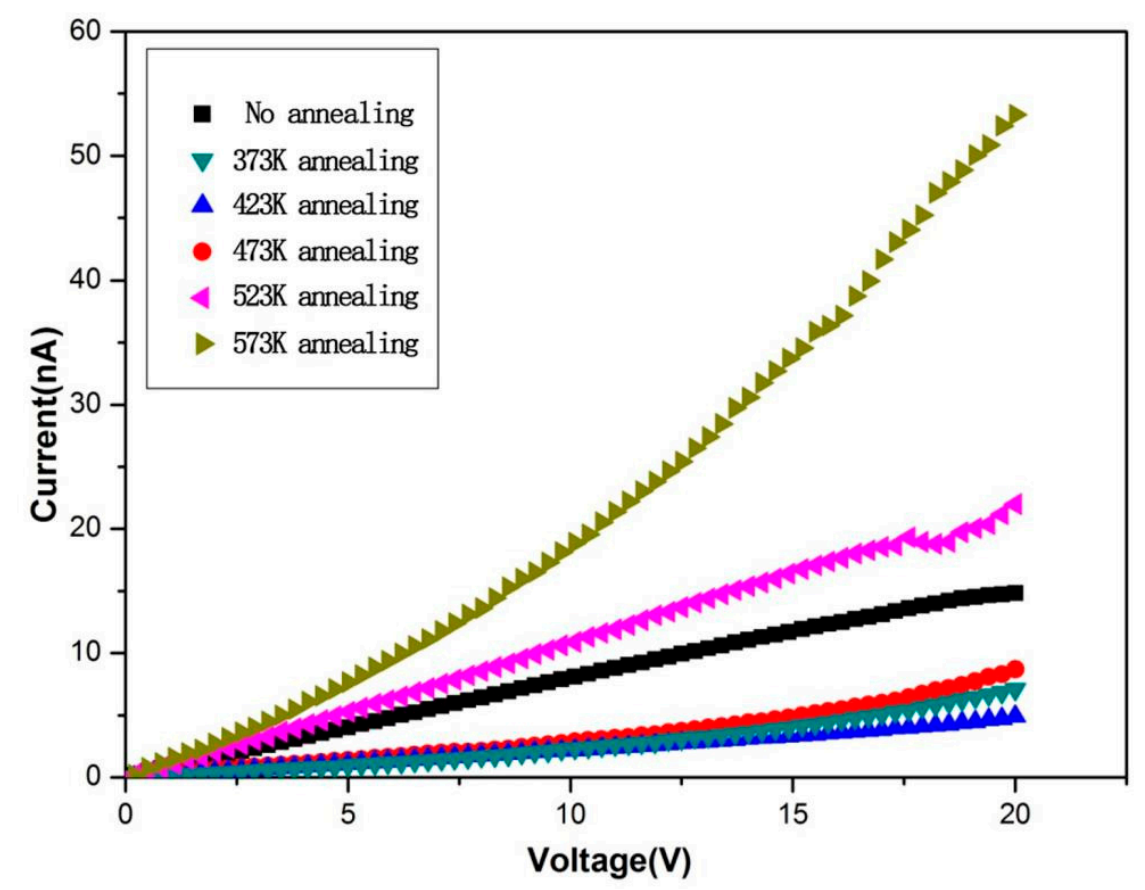

Figure 5. The I-V curves of the Ti-Au/CdZnTe sample after rapid annealing for $4 \mathrm{~min}$ at different temperatures.

Table 3. The I-V curves fitting results of Ti-Au/CdZnTe after rapid annealing at different temperatures for $4 \mathrm{~min}$.

\begin{tabular}{ccccccc}
\hline Annealing Temperature & No Annealing & $\mathbf{3 7 3} \mathbf{K}$ & $\mathbf{4 2 3} \mathbf{~}$ & $\mathbf{4 7 3} \mathbf{~ K}$ & $\mathbf{5 2 3} \mathbf{K}$ & $\mathbf{5 7 3} \mathbf{~}$ \\
\hline Ohmic coefficient b & 0.7274 & 0.7841 & 0.9083 & 0.8315 & 0.7629 & 0.7503 \\
leakage current nA@20 V & 14.82 & 7.07 & 4.91 & 8.68 & 21.98 & 53.32 \\
\hline
\end{tabular}


The $\mathrm{C}-\mathrm{V}$ tests were carried out on $\mathrm{Ti}-\mathrm{Au} / \mathrm{CdZnTe}$ at different annealing temperatures, as shown in Figure 6. When the temperature rises to more than $423 \mathrm{~K}$, the value of capacitance $C$ becomes larger with the negative applied voltage. The barrier height $\Phi_{B}$ is calculated by Equation (1), and is shown in Table 4. It was found that the barrier height of the Ti-Au electrode to CdZnTe decreases with the increase in temperature. As the annealing temperature increases, the concentration of the principal impurity $N_{A}$ gradually increases, and the width of the interface barrier decreases. When the $N_{A}$ value is high enough, the interface is close to heavy doping. Thus, the leakage current increases greatly owing to the tunneling effect, and the barrier height decreases at an elevated temperature.

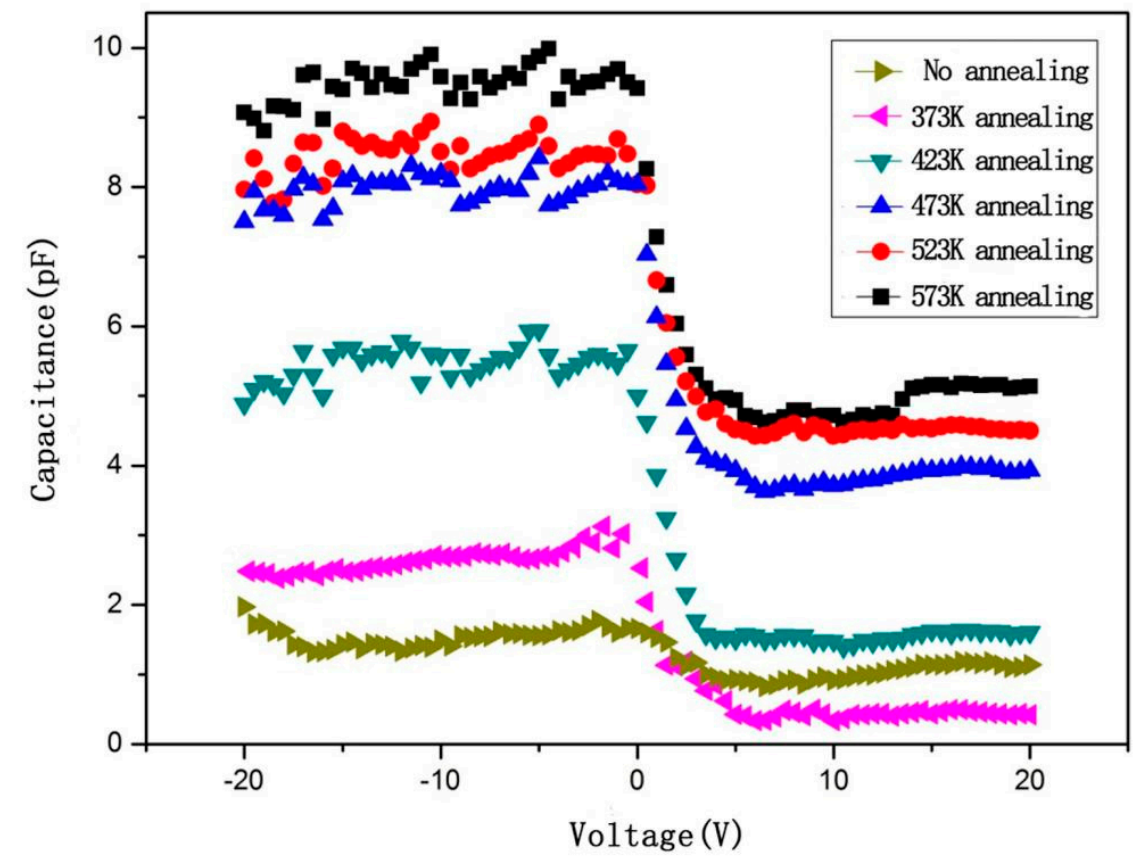

Figure 6. The $\mathrm{C}-\mathrm{V}$ curves of the $\mathrm{Ti}-\mathrm{Au} / \mathrm{CdZnTe}$ sample after rapid annealing for $4 \mathrm{~min}$ at different temperatures.

Table 4. The barrier heights of Ti-Au/CdZnTe after rapid annealing at different temperatures for $4 \mathrm{~min}$.

\begin{tabular}{cc}
\hline $\mathbf{T}$ & $\mathbf{\Phi B}(\mathbf{e V})$ \\
\hline No annealing & 0.932 \\
$373 \mathrm{~K}$ & 0.864 \\
$423 \mathrm{~K}$ & 0.833 \\
$473 \mathrm{~K}$ & 0.812 \\
$523 \mathrm{~K}$ & 0.795 \\
$573 \mathrm{~K}$ & 0.778 \\
\hline
\end{tabular}

The metal electrode deposited on the CdZnTe crystal by the vacuum evaporation method can be easily peeled off in the process of electrode lead owing to its low adhesion, which will affect the subsequent device preparation process and detector performance. Therefore, it is very important to improve the adhesion strength between the electrode and CdZnTe crystal. Theoretically, rapid annealing can enhance the mutual diffusion movement between the electrode and CdZnTe crystal, and thus improve the adhesion between them. A Dage PC2400 microanalyzer was used to measure the adhesion strength of the Ti-Au/CdZnTe electrode, which is treated at different annealing temperatures for $4 \mathrm{~min}$, and the calculated values of adhesion strength are shown in Figure 7. It was found that, from no annealing to $423 \mathrm{~K}$ annealing, the average adhesion strength between Ti-Au and CdZnTe crystals increases from $835.3 \mathrm{Mpa}$ to $1100 \mathrm{Mpa}$, an increase of 30\%. With the annealing temperature above 423 $\mathrm{K}$, the increase of adhesion strength slows down. 


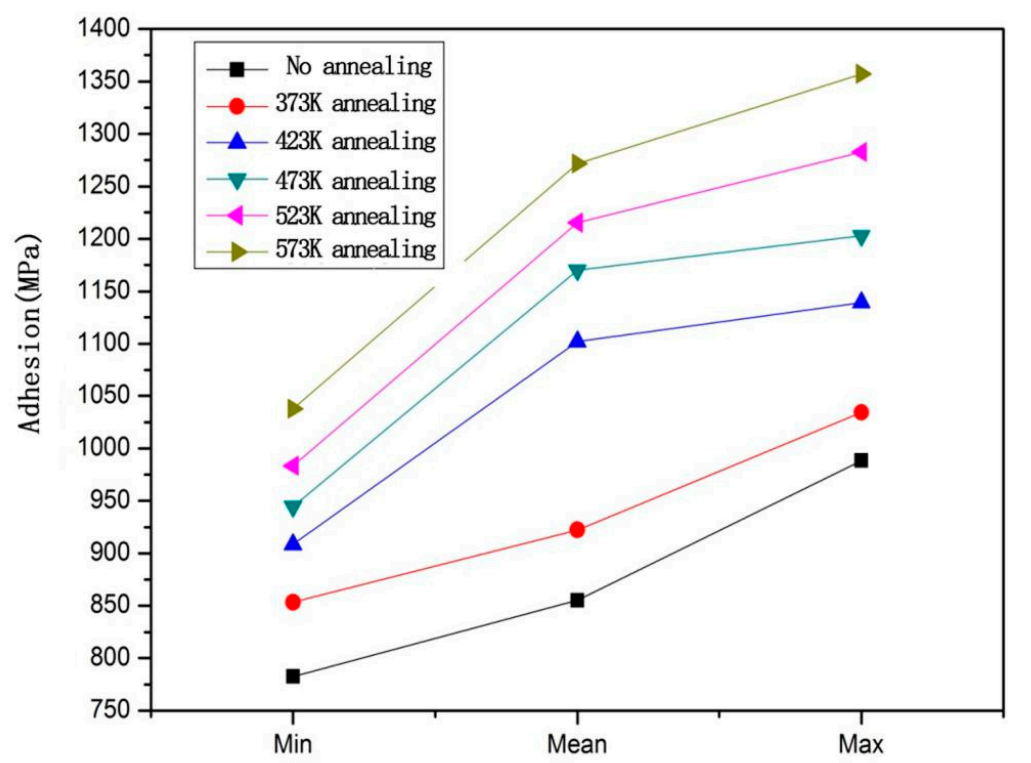

Figure 7. The adhesion between Ti-Au and CdZnTe after rapid annealing at different temperatures for $4 \mathrm{~min}$.

\subsubsection{Effect of Annealing Time on Ti-Au/CdZnTe}

It was proved that the ohmic contact characteristics and adhesion of $\mathrm{Ti}-\mathrm{Au} / \mathrm{CdZnTe}$ were significantly improved after annealing at $423 \mathrm{~K}$. Then, the Ti-Au/CdZnTe sample was rapidly annealed at $423 \mathrm{~K}$ with different annealing times ( $2 \mathrm{~min}, 4 \mathrm{~min}, 6 \mathrm{~min}, 8 \mathrm{~min}$, and $10 \mathrm{~min}$ ) to study the influence of annealing time on the contact performance of Ti-Au/CdZnTe.

Figure 8 shows the $\mathrm{I}-\mathrm{V}$ characteristic curves of the sample after rapid annealing at $423 \mathrm{~K}$ for different times. The I-V characteristic curves are fitted according to the ohmic characteristic formula, and the results are shown in Table 5. It can be seen that, at the annealing temperature of $423 \mathrm{~K}$, when the annealing time reaches $6 \mathrm{~min}$, the leakage current is the lowest and the ohmic contact performance is the best. However, when the annealing time continues to increase to $8 \mathrm{~min}$ and $10 \mathrm{~min}$, the leakage current increases obviously and the ohmic contact performance becomes worse.

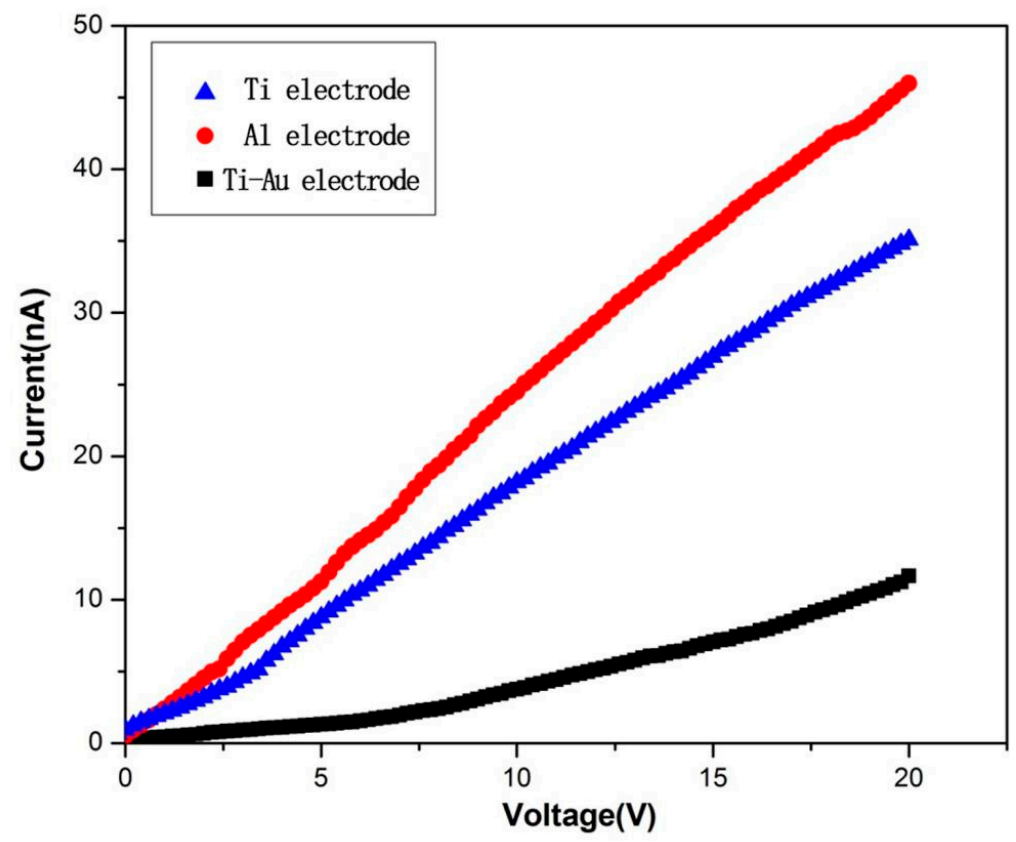

Figure 8. The I-V curves of the Ti-Au/CdZnTe sample annealed at $423 \mathrm{~K}$ for different times. 
Table 5. The I-V curves fitting results of Ti-Au/CdZnTe annealed at $423 \mathrm{~K}$ for different times.

\begin{tabular}{ccccccc}
\hline $\begin{array}{c}\text { Annealing } \\
\text { Time }\end{array}$ & No Annealing & $\mathbf{2}$ min & $\mathbf{4}$ in & $\mathbf{6}$ min & $\mathbf{8}$ min & $\mathbf{1 0}$ min \\
\hline Ohmic coefficient b & 0.7661 & 0.7821 & 0.8783 & 0.91571 & 0.8502 & 0.7133 \\
leakage current $\mathrm{nA}(20 \mathrm{~V})$ & 21.25 & 7.76 & 5.43 & 3.12 & 4.84 & 9.82 \\
\hline
\end{tabular}

The $\mathrm{C}-\mathrm{V}$ measurement was performed on the Ti-Au/CdZnTe sample annealed at $423 \mathrm{~K}$ for different times, and the results are shown in Figure 9. When the Ti-Au/CdZnTe sample is annealed at $423 \mathrm{~K}$ temperature for 4 10 $\mathrm{min}$, the capacitance $C$ changes significantly with the change of external bias voltage V. The potential barrier height $\Phi_{\mathrm{B}}$ is calculated by Equation (1), and the results are shown in Table 6. The barrier height $\Phi_{\mathrm{B}}$ of Ti-Au/CdZnTe decreases after different annealing times. When the annealing time is longer than $6 \mathrm{~min}$, the $\Phi_{\mathrm{B}}$ value changes slowly, which may because of the increase in defects in the crystal and the production of the non-stoichiometric ratio during the long time annealing.

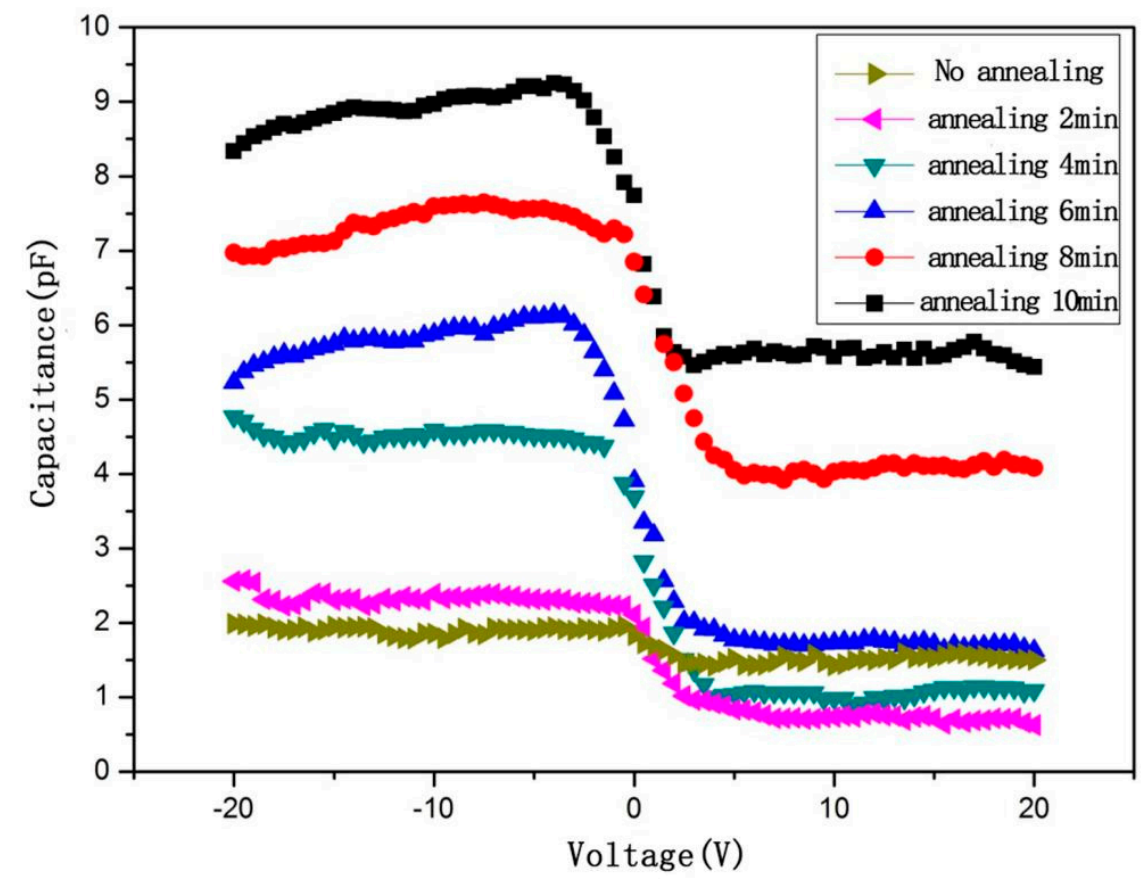

Figure 9. The $\mathrm{C}-\mathrm{V}$ curves of the Ti-Au/CdZnTe samples annealed at $423 \mathrm{~K}$ in an Ar atmosphere for different times.

Table 6. The barrier heights of Ti-Au/CdZnTe annealed at $423 \mathrm{~K}$ for different times.

\begin{tabular}{cc}
\hline $\mathbf{T}$ & $\boldsymbol{\Phi}_{\mathbf{B}}(\mathbf{e V})$ \\
\hline No annealing & 0.941 \\
2 min & 0.912 \\
$4 \mathrm{~min}$ & 0.853 \\
$6 \mathrm{~min}$ & 0.801 \\
$8 \mathrm{~min}$ & 0.787 \\
$10 \mathrm{~min}$ & 0.772 \\
\hline
\end{tabular}

The relationship between the adhesion strength of Ti-Au/CdZnTe and annealing time at $423 \mathrm{~K}$ is compared, as shown in Figure 10. It was found that the adhesion strength between Ti-Au and CdZnTe was $1225 \mathrm{MPa}$ after 6 min annealing, which is $10 \%$ higher than that after 4 min annealing and $50 \%$ higher than that without annealing. Then, when the annealing time is longer than $6 \mathrm{~min}$, the increase rate of the adhesion strength of $\mathrm{Ti}-\mathrm{Au} / \mathrm{CdZnTe}$ decreases. Considering the effect of rapid annealing 
on the leakage current, ohmic characteristics, and adhesion strength of the Ti-Au/CdZnTe electrode, the optimal annealing temperature and time is $423 \mathrm{~K}$ and $6 \mathrm{~min}$, respectively.

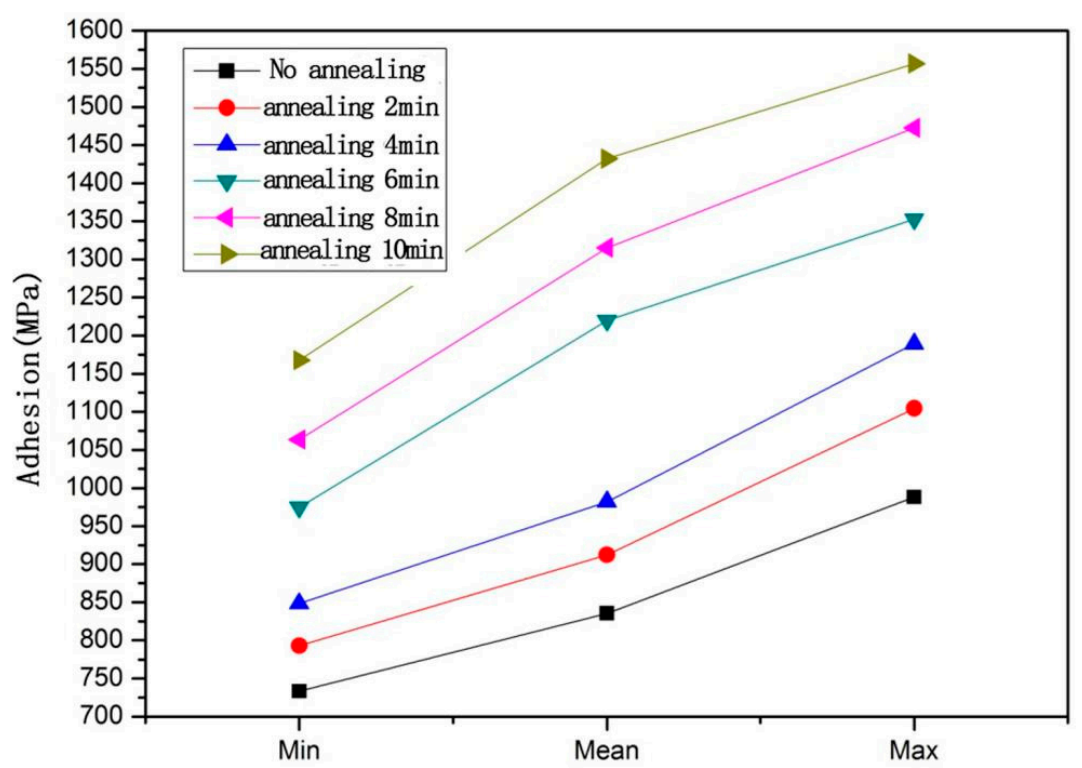

Figure 10. The adhesion between Ti-Au and CdZnTe after rapid annealing at $423 \mathrm{~K}$ for different times.

\section{Conclusions}

$\mathrm{Ti}, \mathrm{Al}$, and Ti-Au electrodes were deposited on the surface of n-type (111) CdZnTe crystal by the vacuum evaporation method. $\mathrm{I}-\mathrm{V}, \mathrm{C}-\mathrm{V}$, and adhesion strength measurements between the various electrodes and CdZnTe crystals were investigated, which indicate that the Ti-Au electrode on CdZnTe shows the lowest leakage current and barrier height, and the highest adhesion strength. Then, the rapid annealing in the Ar atmosphere is used to optimize the contact properties of Ti-Au/CdZnTe. The I-V results show that the Ti-Au/CdZnTe electrode had the best ohmic characteristics after rapid annealing at $423 \mathrm{~K}$ annealing temperature for $6 \mathrm{~min}$, with an ohmic coefficient of 0.9157 and leakage current of $3.12 \mathrm{nA}$ at $20 \mathrm{~V}$. The barrier height of the Ti-Au/CdZnTe electrode is $0.801 \mathrm{eV}$ through rapid annealing for $6 \mathrm{~min}$ at $423 \mathrm{~K}$ annealing temperature, and the adhesion is $1225 \mathrm{MPa}$, which increases by $50 \%$ compared with that without rapid annealing. The results indicate that the Ti-Au composite electrode is a promising ohmic contact to n-type (111) CdZnTe crystal, and the rapid annealing in the Ar atmosphere could improve the ohmic property and adhesion of Ti-Au/CdZnTe contact, with the optimal annealing temperature and time found to be $423 \mathrm{~K}$ and $6 \mathrm{~min}$, respectively.

Author Contributions: Conceptualization, H.S.; methodology, S.W.; formal analysis, J.H.; investigation, H.S.; data curation, Z.S. and J.M.; writing-original draft preparation, H.S.; writing-review and editing, S.W.; supervision, L.W.; project administration, J.Z. All authors have read and agree to the published version of the manuscript.

Funding: This research was funded by National Natural Science Foundation of China, grant number 51472155, $11675099,11505109$.

Acknowledgments: The authors are grateful to the National Natural Science Foundation of China under grant (No. 51472155, 11675099, 11505109).

Conflicts of Interest: The authors declare no conflict of interest.

\section{References}

1. Takahashi, T.; Watanabe, S. Recent progress in CdTe and CdZnTe detectors. IEEE Trans. Nucl. Sci. 2001, 48, 950-959. [CrossRef]

2. Limousin, O. New trends in CdTe and CdZnTe detectors for X-and gamma-ray applications. Methods Phys. Res. A 2003, 504, 24-37. 
3. Amman, M.; Lee, J.S.; Luke, P.N.; Chen, H.; Awadalla, S.A.; Redden, R.; Bindley, G. Evaluation of THM-grown CdZnTe material for large-volume gamma-ray detector applications. IEEE Trans. Nucl. Sci. 2009, 56, 795-799. [CrossRef]

4. Verger, L.; Bonnefoy, J.P.; Glasser, F.; Ouvrier-Buffet, P. New developments in CdTe and CdZnTe detectors for $\mathrm{X}$ and $\gamma$-ray applications. J. Electron. Mater. 1997, 26, 738-744. [CrossRef]

5. Awadalla, S.A.; Mackenzie, J.; Chen, H.; Redden, B.; Bindley, G.; Duff, M.C.; Burger, A.; Groza, M.; Buliga, V.; Bradley, J.P.; et al. Characterization of detector-grade CdZnTe crystals grown by traveling heater method (THM). J. Cryst. Growth 2010, 312, 507-513. [CrossRef]

6. Szeles, C. CdZnTe and CdTe materials for X-ray and gamma ray radiation detector applications. Stat. Sol. 2004, 241, 783-790. [CrossRef]

7. Duff, M.C.; Hunter, D.B.; Burger, A.; Groza, M.; Buliga, V.; Bradley, J.P.; Graham, G.; Dai, Z.R.; Teslich, N.; Black, D.R.; et al. Characterization of heterogeneities in detector-grade CdZnTe crystals. J. Mater. Res. 2009, 24, 1361-1367. [CrossRef]

8. Yu, P.; Chen, Y.; Li, W.; Liu, W.; Liu, B.; Yang, J.; Ni, K.; Luan, L.; Zheng, J.; Li, Z.; et al. Study of Detector-Grade CdMnTe: In Crystals Obtained by a Multi-Step Post-Growth Annealing Method. Crystals 2018, 8, 387. [CrossRef]

9. Bolotnikov, A.E.; Camarda, G.C.; Carini, G.A.; Fiederle, M.; Li, L.; McGregor, D.S.; McNeil, W.; Wright, G.W.; James, R.B. Performance characteristics of Frisch-ring CdZnTe detectors. IEEE Trans. Nucl. Sci. 2006, 53, 607-614. [CrossRef]

10. Chen, H.; Awadalla, S.A.; Iniewski, K.; Lu, P.H.; Harris, F.; Mackenzie, J.; Hasanen, T.; Chen, W.; Redden, R.; Bindley, G. Characterization of large cadmium zinc telluride crystals grown by traveling heater method. J. Appl. Phys. 2008, 103, 014903. [CrossRef]

11. Sun, J.; Fu, L.; Nie, Z.; Shi, Y.; Li, Y. The effect of chemical polishing on the interface structure and electrical property of $\mathrm{Au} / \mathrm{Cd}_{0.9} \mathrm{Zn}_{0.1}$ Te contact. Appl. Phys. A 2014, 115, 1309-1316. [CrossRef]

12. Tari, S.; Aqariden, F.; Chang, Y.; Ciani, A.; Grein, C.; Li, J.; Kioussis, N. Structural and electronic properties of gold contacts on CdZnTe with different surface finishes for radiation detector applications. J. Electron. Mater. 2014, 43, 2978-2983. [CrossRef]

13. Yang, L.; Min, J.; Liang, X.; Liu, Z.; Lin, Y.; Zhang, J.; Wang, L.; Shen, Y.; Zhang, Y.; Li, M. Investigation on the contact interface of Au/Zn on CdZnTe (111) B surface. Mater. Sci. Semicond. Process. 2017, 67, 175-180. [CrossRef]

14. Ling, L.; Zhang, J.; Zhao, S.; Zhang, D.; Zhang, J.; Shi, H.; Tang, K.; Liang, X.; Huang, J.; Min, J.; et al. Surface analysis and electrical measurement of the ohmic contact on p-CdZnTe (111) B face with Au/Cd composite electrode. Mater. Sci. Semicond. Process. 2019, 98, 90-94. [CrossRef]

15. Bell, S. Fabrication and Characterisation of Gold Contacts on CdZnTe Radiation Detectors; University of Surrey: Guildford, UK, 2015.

16. Liang, X.Y.; Min, J.H.; Chen, J.; Wang, D.; Li, H.; Wang, Y.; Wang, L.J.; Zhang, J.J. Metal/semiconductor contacts for schottky and photoconductive CdZnTe detector. Phys. Procedia 2012, 32, 545-550. [CrossRef]

17. Talasek, R.T. Electrochemistry of Semiconductors and Electronics; McHardy, J., Bahir, F.G., Eds.; Noyes Publications: Park Ridge, IL, USA, 1992; p. 127.

18. Sang, W.; Wei, J.; Qi, Z.; Wanwan, L.; Jiahua, M.; Jianyong, T.; Yongbiao, Q. Primary study on the contact degradation mechanism of CdZnTe detectors. Nucl. Instrum. Methods Phys. Res. A 2004, 527, 487-492. [CrossRef]

19. Xie, J.; Liu, Y.; Wang, C. Au Film Electrodes on CdZnTe Surface: Preparation and Ohmic Contact Property. J. Inog. Mater. 2018, 33, 273-278.

(C) 2020 by the authors. Licensee MDPI, Basel, Switzerland. This article is an open access article distributed under the terms and conditions of the Creative Commons Attribution (CC BY) license (http://creativecommons.org/licenses/by/4.0/). 